\title{
Estado nutricional e condições ambientais e de saúde de crianças Pataxó, Minas Gerais, Brasil
}

\author{
Nutritional status and environmental and health \\ conditions of Pataxó indigenous children, Minas \\ Gerais State, Brazil
}

\section{Estado nutricional y condiciones ambientales y de salud de niños Pataxó, Minas Gerais, Brasil}

\author{
Anabele Pires Santos 1 \\ Camila Medeiros da Silva Mazzeti 2 \\ Maria do Carmo Pinho Franco 1 \\ Nicole Louise Gonzaga Oliveira Santos 3 \\ Wolney Lisboa Conde 2 \\ Maurício Soares Leite 4 \\ Adriano Marçal Pimenta 3 \\ Lenice de Castro Mendes Villela 3 \\ Teresa Gontijo de Castro 5
}

\section{Resumo}

Descrever o estado nutricional e as condições ambientais e de saúde das crianças Pataxó de cinco aldeias de Minas Gerais, Brasil. O estado nutricional foi classificado com base no peso e estatura/comprimento, tendo como referência o padrão de crescimento da Organização Mundial da Saúde. Questionários baseados no I Inquérito Nacional de Saúde e Nutrição dos Povos Indígenas foram utilizados para a avaliação das condições ambientais dos domicílios e de saúde dos menores de cinco anos. Dos 70 menores de dez anos avaliados (93,3\%), 34 tinham menos de cinco anos. Não se observaram déficits nutricionais e sobrepeso foi registrado para 11,4\% das crianças. A maioria das crianças (74,3\%) vivia em domicílios com energia elétrica, 95\% em domicílios com latrina/sanitário e 52,9\% lançavam dejetos em fossa séptica. A realização de seis ou mais consultas de pré-natal foi reportada por $82,4 \%$ das mães dos menores de cinco anos, e 91,2\% iniciaram o pré-natal no primeiro trimestre de gestação. Dentre as causas de internações hospitalares nos últimos 12 meses (23,5\%), somente uma foi devido à diarreia e nenhuma por causa de infecções respiratórias. Foram verificadas coberturas universais para a maioria das vacinas avaliadas. A inexistência de déficits nutricionais entre as crianças Pataxó pode estar associada às melhores condições de habitação, saneamento e cobertura das ações básicas de saúde infantil quando comparadas às condições verificadas no I Inquérito Nacional de Saúde e Nutrição dos Povos Indígenas e de estudos pontuais. Este trabalho pretende subsidiar discussões $e$ ações que visem a melhorias do estado nutricional infantil dos indígenas no Brasil.

Saúde de Populações Indígenas; Antropometria; Índios Sul-Americanos; Estado Nutricional

\author{
Correspondência \\ T. G. Castro \\ Centre for Longitudinal Research, University of Auckland. \\ Tamaki Campus, 261 Morrin Road, Glen Innes, Auckland \\ 1743, New Zealand. \\ t.castro@auckland.ac.nz \\ 1 Escola Paulista de Medicina, Universidade Federal de São \\ Paulo, São Paulo, Brasil. \\ 2 Faculdade de Saúde Pública, Universidade de São Paulo, São \\ Paulo, Brasil. \\ 3 Escola de Enfermagem, Universidade Federal de Minas Gerais, \\ Belo Horizonte, Brasil. \\ 4 Departamento de Nutrição, Universidade Federal de Santa \\ Catarina, Florianópolis, Brasil. \\ 5 Centre for Longitudinal Research, University of Auckland, \\ Auckland, New Zealand.
}




\section{Introdução}

No Brasil, apesar da realização do I Inquérito Nacional de Saúde e Nutrição dos Povos Indígenas em 2008/2009 1 e do aumento das publicações na última década acerca do estado nutricional infantil com base em estudos específicos 2,3, a quantidade de informações geradas ainda é desproporcional ao conhecimento produzido sobre a temática para as crianças não indígenas. Trata-se de informações fundamentais para subsidiar intervenções que visem a reduzir as iniquidades observadas para o segmento 4 . Este estudo objetiva descrever o estado nutricional e as condições ambientais e de saúde das crianças Pataxó de cinco aldeias de Minas Gerais, Brasil.

\section{Métodos}

Na década de 1940, a área habitada pelos Pataxó no litoral do sul baiano foi delimitada para a criação do Parque Nacional do Monte Pascoal, o que repercutiu, em 1951, em um conflito ("Fogo de 51") que envolveu a intervenção violenta da polícia local e culminou na fuga de um grupo de Pataxó à procura de abrigo seguro na região de Carmésia, Minas Gerais 5. Este é um estudo transversal, conduzido entre outubro e dezembro de 2011 (à época, os Pataxó viventes em Minas Gerais correspondiam a 418 indivíduos, distribuídos em sete aldeias - Figura 1). Foram estudadas as crianças residentes nas cinco aldeias Pataxó cujas lideranças aceitaram a participação: Cinta Vermelha, Serra do Candonga, Gerú Tucumã, Fazenda Guarani e Imbiriçu.

Realizou-se avaliação nutricional dos menores de dez anos e medidas de peso (quilogramas) e estatura/comprimento (centímetros), em duplicata, foram coletadas de acordo com os protocolos da Organização Mundial da Saúde (OMS) 6. Entre os menores de dois anos, peso e comprimento foram coletados, respectivamente, utilizando-se balança digital portátil Beurer (capacidade de $20 \mathrm{~kg}$ e precisão de 10g; Beurer GmbH, Ulm, Alemanha) e infantômetro portátil Alturaexata (extensão de 1,2m e precisão de 1mm; Alturaexata, Belo Horizonte, Brasil). Crianças de dois anos ou mais tiveram peso e altura coletados, respectivamente, por balança digital portátil Marte (capacidade de $150 \mathrm{~kg}$ e precisão de 50g; Marte Científica, São Paulo, Brasil) e estadiômetro Alturaexata (extensão de $2 \mathrm{~m}$ e precisão de $1 \mathrm{~mm}$; Alturaexata, Belo Horizonte, Brasil). Com o auxílio dos programas WHO-Anthro (http:// www.who.int/childgrowth/software/en/) e WHO-Anthro-Plus (http://www.who.int/growthref/ tools/en/) foram calculados os desvios padronizados (z) dos índices: índice estatura para idade (E/I), índice peso para idade (P/I) e IMC/I (índice de massa corporal para idade). A classificação do estado nutricional foi baseada nas curvas de crescimento da OMS 7,8.

Questionários valendo-se do I Inquérito Nacional de Saúde e Nutrição dos Povos Indígenas 9 (que denominaremos Inquérito de Saúde Indígena Nacional a partir daqui) foram utilizados para a avaliação das condições ambientais e de saneamento dos domicílios onde viviam os menores de dez anos (respondidos pelo responsável pelo domicílio) e para a avaliação dos indicadores de saúde dos menores de cinco anos (respondidos pelas mães). Coberturas vacinais entre os menores de cinco anos foram avaliadas de acordo com a idade e com o calendário vacinal nacional estabelecido para as populações indígenas à época 10.

O estudo foi aprovado em todas as instâncias requeridas pela Fundação Nacional do Índio (FUNAI, processo no 1141/2008) 11. Foram obtidos os Termos de Consentimento Livre e Esclarecido dos responsáveis. Conduziu-se as análises no programa IBM SPSS versão 17.0. (IBM Corp., Armonk, Estados Unidos).

\section{Resultados}

Dentre os 75 menores de dez anos residentes nas aldeias, 70 (93,3\%) fizeram parte do estudo, sendo 34 $(48,5 \%)$ menores de cinco anos. Todos os chefes das famílias dos 37 domicílios onde viviam os menores de dez anos e todas as mães destes menores responderam aos questionários aplicados.

Déficits estatural e de $\mathrm{P} / \mathrm{I}$ foram inexistentes em qualquer faixa etária. Sobrepeso classificado pelo IMC/I acometeu 11,4\% dos menores de dez anos (2,9\% dos menores de cinco anos e 19,4\% das 


\section{Figura 1}

Localização das sete aldeias Pataxó no Estado de Minas Gerais, Brasil, outubro de 2011.

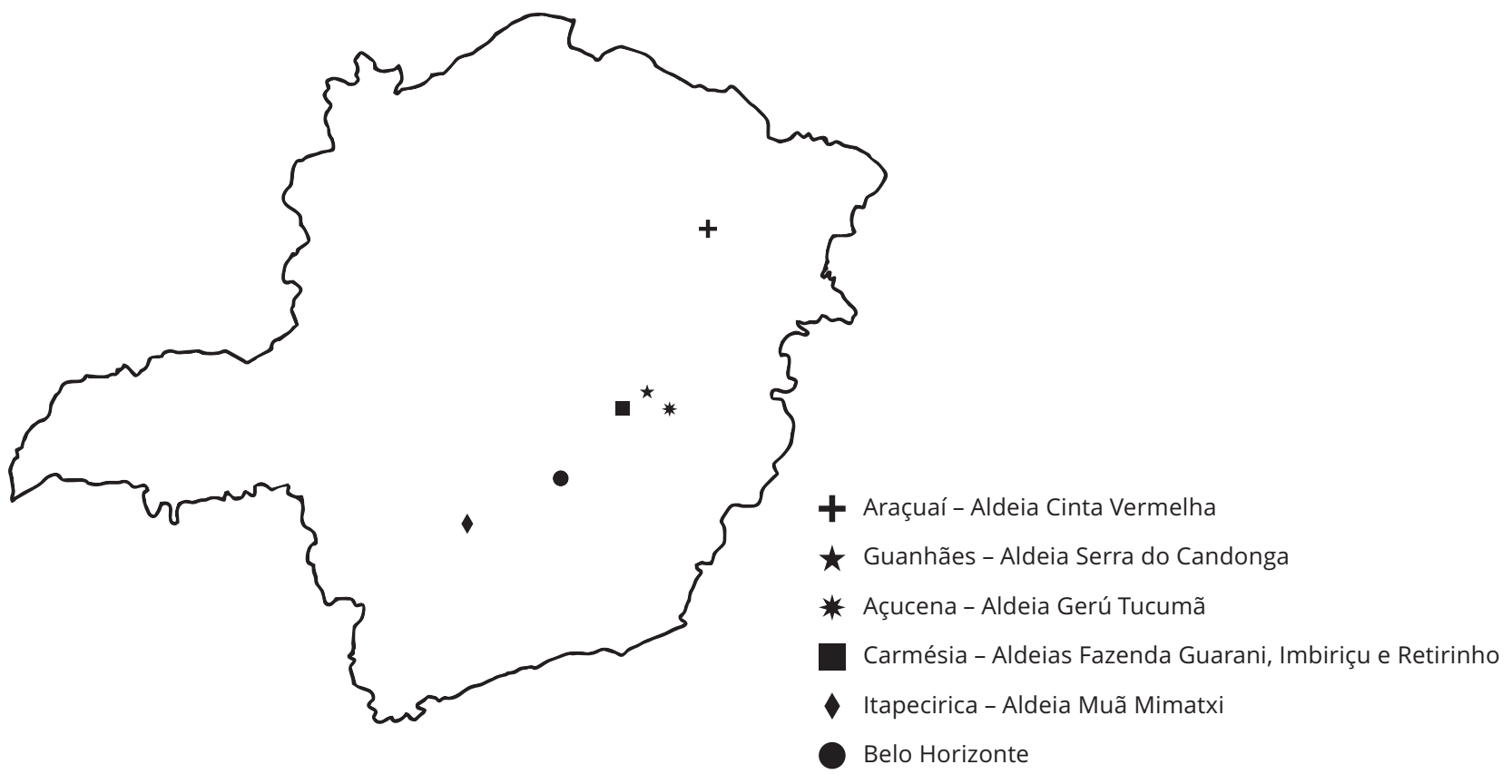

$\begin{array}{llllll}0 & 60 & 20 & 240 & 360 & 480 \mathrm{~km}\end{array}$

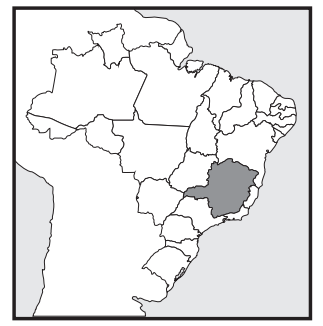

Fonte: elaboração própria.

crianças com cinco ou mais anos) e peso elevado para idade (P/I) acometeu 5,7\% dos menores de dez anos (ausentes casos entre os menores de cinco anos e 11,1\% das crianças com cinco ou mais anos).

A maioria dos menores de dez anos vivia em casas com energia elétrica e com piso de madeira, cerâmica ou cimento. Aproximadamente metade vivia em domicílios com telhado de telha de barro, 94\% em residências com latrina/sanitário e metade vivia em domicílios que lançavam dejetos em fossa séptica. Metade das crianças vivia em domicílios cujo lixo era coletado pelo serviço de limpeza local das prefeituras, e para 32,9\% a água utilizada para beber vinha da rede pública ou cacimbas (poço de água potável). Independentemente da origem da água, a filtragem da água para beber foi reportada para 60\% das crianças (Tabela 1).

Para a maioria dos menores de cinco anos as mães possuíam a Carteira de Pré-natal (Ministério da Saúde), e o acompanhamento começou no primeiro trimestre de gestação, com seis ou mais consultas realizadas durante a gravidez. Quase todas as crianças $(n=33 / 34)$ nasceram em hospitais, $14,7 \%$ foram pré-termos e coberturas quase universais foram registradas para as imunizações avaliadas. Todas as crianças tinham a Caderneta da Criança (Ministério da Saúde), mas registro recente 
Tabela 1

Domicílios dos Pataxó menores de dez anos: características de construção, de saneamento, destino de dejetos e lixo e origem da água para beber. Minas Gerais, Brasil, 2011.

\begin{tabular}{|c|c|}
\hline Variáveis/Categorias & n (\%) \\
\hline \multicolumn{2}{|l|}{ Tipo de piso } \\
\hline Terra & $11(15,7)$ \\
\hline Madeira, cerâmica ou cimento & $58(82,9)$ \\
\hline Outro & $1(1,4)$ \\
\hline \multicolumn{2}{|l|}{ Tipo de parede } \\
\hline Tijolo & $37(52,9)$ \\
\hline Taipa/Barro & $32(45,7)$ \\
\hline Outro & $1(1,4)$ \\
\hline \multicolumn{2}{|l|}{ Tipo de telhado/cobertura } \\
\hline Telha de barro & $40(57,1)$ \\
\hline Telha de zinco ou amianto & $29(41,4)$ \\
\hline Outro & $1(1,4)$ \\
\hline \multicolumn{2}{|l|}{ Energia elétrica } \\
\hline Sim & $52(74,3)$ \\
\hline Não & $18(25,7)$ \\
\hline \multicolumn{2}{|l|}{ Local onde os moradores defecam } \\
\hline Latrina/Sanitário de uso do domicílio & $59(84,3)$ \\
\hline Latrina/Sanitário de uso coletivo & $7(10,0)$ \\
\hline Outros & $4(5,7)$ \\
\hline \multicolumn{2}{|l|}{ Se houver latrina/sanitário, destino dos dejetos } \\
\hline Fossa séptica & $37(52,9)$ \\
\hline Fossa rudimentar/rasa & $12(17,1)$ \\
\hline Vala, rio, lago/açude & $17(24,3)$ \\
\hline Não se aplica & $4(5,7)$ \\
\hline \multicolumn{2}{|l|}{ Destino do lixo } \\
\hline Coletado por serviço de limpeza & $35(50,0)$ \\
\hline Enterrado, jogado ou queimado na aldeia & $33(47,2)$ \\
\hline Outros & $2(2,8)$ \\
\hline \multicolumn{2}{|l|}{ Origem da água utilizada para beber } \\
\hline Rede pública/municipal & $6(8,6)$ \\
\hline Cacimba & $17(24,3)$ \\
\hline Poço, mina d'água, fonte protegida, rio, lago, igarapé, açude ou outro & $47(67,1)$ \\
\hline \multicolumn{2}{|l|}{ No domicílio, a água usada para beber é } \\
\hline Filtrada & $42(60,0)$ \\
\hline Não realiza tratamento na água para beber & $28(40,0)$ \\
\hline
\end{tabular}

Nota: $\mathrm{N}=70$ crianças distribuídas em 37 domicílios.

de pesagem foi verificado somente para uma criança. A maioria foi suplementada com megadose de vitamina A em algum momento da vida, porém pouco mais da metade havia recebido a suplementação nos últimos seis meses anteriores à pesquisa. $\mathrm{O}$ uso de sulfato ferroso nos últimos três meses antes da pesquisa entre as crianças de 6 a 18 meses foi de $28,6 \%$ (dois casos). Internação hospitalar nos últimos 12 meses foi reportada para quase um quarto das crianças, mas somente uma internação foi devido à diarreia e nenhuma por causa de infecções respiratórias. Para 17,6\% das crianças (seis casos) foi reportada a ocorrência de diarreia na última semana anterior à pesquisa, e tosse para cerca de $35,3 \%$ (12 casos) (Tabela 2$)$. 
Tabela 2

Pataxó menores de cinco anos: características do pré-natal e nascimento, situação vacinal, acompanhamento do crescimento e desenvolvimento, suplementação de sulfato ferroso e vitamina A, e internações e morbidade referida. Minas Gerais, Brasil, 2011.

\begin{tabular}{|c|c|}
\hline Variáveis/Categorias & n (\%) \\
\hline Mãe possuía Carteira de Pré-natal (Ministério da Saúde) & $29(85,3)$ \\
\hline Primeira consulta de pré-natal no primeiro trimestre & $31(91,2)$ \\
\hline Número de consultas pré-natal $\geq 6$ & $28(82,4)$ \\
\hline \multicolumn{2}{|l|}{ Características do pré-natal } \\
\hline Feito por médico ou enfermeiro & $34(100,0)$ \\
\hline Pressão arterial materna foi medida & $33(97,1)$ \\
\hline Altura uterina foi medida & $33(97,1)$ \\
\hline Coração do bebê foi auscultado & $34(100,0)$ \\
\hline Mãe foi medida e pesada & $33(97,1)$ \\
\hline Mama materna foi examinada & $26(76,5)$ \\
\hline Criança nasceu no hospital & $33(97,1)$ \\
\hline Criança possuía Caderneta da Criança (Ministério da Saúde) & $34(100,0)$ \\
\hline Peso ao nascer $<2.500 \mathrm{~g}$ & $2(5,9)$ \\
\hline Idade gestacional < 37 semanas & $5(14,7)$ \\
\hline Criança possuía registro de vacinas no cartão da criança & $33(97,1)$ \\
\hline \multicolumn{2}{|l|}{ Vacinas recebidas } \\
\hline BCG & $34(100,0)$ \\
\hline Hepatite B (1 $\underline{a}$ dose ao nascer) & $34(100,0)$ \\
\hline Poliomielite & $33(100,0)$ \\
\hline Tetravalente & $15(100,0)$ \\
\hline DTP & $25(96,2)$ \\
\hline Tríplice viral & $27(100,0)$ \\
\hline Influenza & $28(96,6)$ \\
\hline Rotavírus & $27(90,0)$ \\
\hline Pentavalente & $26(100,0)$ \\
\hline Criança possuía registro de pesagem na Caderneta da Criança & $30(88,2)$ \\
\hline Criança possuía registro de pesagem nos últimos 30 dias & $3(8,8)$ \\
\hline Criança possuía registro do desenvolvimento psicomotor na caderneta da criança & $1(2,9)$ \\
\hline Criança foi suplementada com megadose de vitamina A alguma vez & $28(82,4)$ \\
\hline Criança foi suplementada com a megadose de vitamina A nos últimos 6 meses * & $19(55,9)$ \\
\hline Crianças de 6 a 18 meses que fizeram uso de sulfato ferroso nos últimos 3 meses & $2(28,6)$ \\
\hline Criança foi internada nos últimos 12 meses & $8(23,5)$ \\
\hline Dentre os internados, internação foi devido à diarreia & $1(12,5)$ \\
\hline Dentre os internados, internação foi devido à IRA/pneumonia & $0(0,0)$ \\
\hline Dentre os internados, internação foi devido à tuberculose & $0(0,0)$ \\
\hline Criança teve diarreia na última semana & $6(17,6)$ \\
\hline Criança teve tosse na última semana & $12(35,3)$ \\
\hline
\end{tabular}

BCG: Bacillus Calmette-Guérin; DTP: difteria, tétano e pertussis; IRA: insuficiência respiratória aguda. Nota: $\mathrm{N}=34$.

* Avaliado entre as sete crianças com idades entre 6 e 18 meses. 


\section{Discussão}

Este é o primeiro trabalho a descrever o estado nutricional e as condições ambientais e de saúde de crianças Pataxó viventes em Minas Gerais e a apontar para a inexistência de déficits antropométricos, contrastando grandemente com as prevalências expressivas de déficit estatural e de P/I reportadas pelo Inquérito de Saúde Indígena Nacional 12 e para diferentes etnias em estudos pontuais (com valores oscilando de 5,6\% a 55\% para déficit estatural e de 1,4\% a $45 \%$ para déficit de P/I) 2 .

Os determinantes da desnutrição infantil são multicausais, inter-relacionados e atuam em níveis de determinação imediatos, intermediários e distais, tais como as condições ambientais dos domicílios, cobertura e qualidade do abastecimento de água e de coleta de esgoto e lixo, assistência à saúde materno-infantil e imunização infantil 13. Ressalta-se que, embora aldeados, os Pataxó encontram-se próximos a centros urbanos, o que logisticamente os aproxima também de um conjunto de serviços básicos e de atenção à saúde com potencial impacto positivo sobre indicadores de saúde e nutrição infantil. Comparado aos estudos disponíveis realizados com indígenas $2,3,9,12,14$, para todos os indicadores de saneamento e habitação, e para alguns dos indicadores de assistência materno-infantil, melhores condições foram verificadas entre os Pataxó e poderiam explicar, em parte, a ausência de desnutrição entre as crianças. Dentre os indicadores de assistência à saúde materno-infantil destacam-se: maior cobertura de pré-natal realizada no primeiro trimestre gestacional e com o mínimo de seis consultas realizadas (de acordo com o preconizado pelo Ministério da Saúde 15), maiores coberturas para as imunizações avaliadas e reduzido número de admissões hospitalares devido à diarreia (um caso) e infecção respiratória (nenhum caso).

O relevante percentual de sobrepeso registrado entre as crianças Pataxó está em consonância com o reportado pela literatura da saúde indígena brasileira 2,3, e seus possíveis determinantes têm se relacionado às drásticas mudanças nos padrões de assentamento e estratégias de subsistência, tais como modificações de práticas alimentares nativas 3 .

As observações do presente estudo pretendem subsidiar discussões e ações que visem a melhorias do estado nutricional de crianças indígenas no Brasil.

\section{Colaboradores}

A. P. Santos, M. C. P. Franco e A. M. Pimenta participaram da análise e redação do manuscrito. C. M. S. Mazzeti, N. L. G. O. Santos participaram da coleta de informações, análise e redação do manuscrito. W. L. Conde, M. S. Leite e L. C. M. Villela participaram da elaboração do estudo, análise e redação do manuscrito. T. G. Castro participou da elaboração do estudo, coleta de informações, análise e redação do manuscrito. Todos os autores aprovaram a versão final a ser publicada.

\section{Agradecimentos}

Ao povo Pataxó das aldeias estudadas, pela abertura e receptividade, e ao Distrito Sanitário Especial Indígena, Minas Gerais/Espírito Santo pelas informações providas. À Fundação Amparo à Pesquisa de Minas Gerais (FAPEMIG); Coordenação de Aperfeiçoamento de Pessoal de Nível Superior (Capes). 


\section{Referências}

1. Coimbra Jr. CEA, Santos RV, Welch JR, Cardoso AM, de Souza MC, Garnelo L, et al. The First National Survey of Indigenous People's Health and Nutrition in Brazil: rationale, methodology, and overview of results. BMC Public Health 2013; 13:52.

2. Castro TG, Mazzetti CMS, Mazzuccheti L, Gimeno SGA. Alimentação e nutrição de povos indígenas brasileiros. In: Cardoso MA, organizador. Nutrição em saúde coletiva. São Paulo: Editora Atheneu; 2014. p. 91-103.

3. Leite MS. Nutrição e alimentação em saúde indígena: notas sobre a importância e a situação atual. In: Garnelo L, Pontes AL, organizadores. Saúde indígena: uma introdução ao tema. Brasília: Secretaria de Educação Continuada, Alfabetização, Diversidade e Inclusão, Ministério da Educação; 2012. p. 156-83. (Coleção Educação para Todos).

4. Santos RV, Coimbra Jr. CEA. Cenários e tendências da saúde e da epidemiologia dos povos indígenas no Brasil. In: Coimbra Jr., CEA, Santos RV, Escobar AL, organizadores. Epidemiologia e saúde dos povos indígenas no Brasil. Rio de Janeiro: Editora Fiocruz; 2003. p. 13-47.

5. Carvalho MR. O Monte Pascoal, os índios Pataxó e a luta pelo reconhecimento étnico. Caderno CRH 2009; 22: 507-21.

6. World Health Organization. WHO child growts standards. Training course on child growth assessment. Geneva: World Health Organization; 2008.

7. World Health Organization. WHO child growth standards. Length/height-for-age, weight-forage, weight-for-length, weight-for height and body mass index-for-age: methods and development. Geneva: World Health Organization; 2006.

8. Onis M, Onyango AW, Borghi E, Siyam A, Nishida C, Siekmann J. Development of a WHO growth reference for school-aged children and adolescents. Bull World Health Organ 2007; 85:660-7.
9. Associação Brasileira de Pós-graduação em Saúde Coletiva. Inquérito Nacional de Saúde e Nutrição dos Povos Indígenas: relatório final (análise dos dados). Rio de Janeiro: Associação Brasileira de Pós-graduação em Saúde Coletiva; 2009.

10. Ministério da Saúde. Portaria no 1.946, de 19 de julho de 2010. Institui, em todo o território nacional, o Calendário de Vacinação para os Povos Indígenas. Diário Oficial da União 2010; 20 jul.

11. Fundação Nacional do Índio. Instrução normativa no 01/PRESI, de 29 de novembro de 1995. Brasília: Fundação Nacional do Índio; 1995.

12. Horta BL, Santos RV, Welch JR, Cardoso AM, Santos JV, Assis AMO, et al. Nutritional status of indigenous children: findings from the First National Survey of Indigenous People's Health and Nutrition in Brazil. Int J Equity in Health 2013; 12:23.

13. United Nations Children's Fund. The state of the world's children: focus on nutrition. New York: United Nations Children's Fund; 1998.

14. Coimbra Jr. CEA. Saúde e povos indígenas no Brasil: reflexões a partir do I Inquérito Nacional de Saúde e Nutrição Indígena. Cad Saúde Pública 2014; 30:855-9.

15. Ministério da Saúde. Pré-natal e puerpério: atenção qualificada e humanizada. Manual técnico. Brasília: Ministério da Saúde; 2006. (Série A. Normas e Manuais Técnicos). (Série Direitos Sexuais e Direitos Reprodutivos, 5). 


\section{Abstract}

To describe the nutritional status and the environmental and health conditions of the Pataxó children from five villages of Minas Gerais State, Brazil. Among the under 10 years old, weight and height/length were classified according to the growth references of World Health Organization. Questionnaires evaluating environmental conditions of the households and health conditions of under 5 years old were based in the First National Survey of Indigenous People's Health and Nutrition. Among the 70 children evaluated (93.3\%), 34 were under 5 years old. Nutritional deficits were not observed and overweight was registered for $11.4 \%$ of the children. Most of the children (74.3\%) lived in households with electric energy, 95\% in households with toilets and $52.9 \%$ in households that threw waste in septic tanks. Six or more antenatal appointments were reported by $82.4 \%$ of the mothers of the under five years old and $91.2 \%$ started the antenatal appointments within the first trimester of pregnancy. Among the causes of hospitalizations in the previous 12 months (23.5\%), only one was due to diarrhea and none to respiratory infection. Universal coverage was observed for the majority of the vaccines. The absence of nutritional deficits among the Pataxó children may be associated to better housing and sanitation conditions and coverage of basic childhood health actions when compared to the conditions reported by the First National Survey of Indigenous People's Health and Nutrition and related studies with other specific indigenous peoples. The current study aims to back discussions and measures to improve the nutritional status of indigenous children in Brazil.

Health of Indigenous Peoples; Anthropometry; South American Indians; Nutritional Status

\section{Resumen}

El objetivo de este trabajo fue describir el estado nutricional, así como las condiciones ambientales $y$ de salud de niños Pataxó, procedentes de cinco aldeas de Minas Gerais, Brasil. El estado nutricional se clasificó en base al peso y estatura/longitud, teniendo como referencia el patrón de crecimiento de la Organización Mundial de la Salud. Se utilizaron cuestionarios basados en la I Encuesta Nacional de Salud y Nutrición de los Pueblos Indígenas para la evaluación de las condiciones ambientales de los domicilios y de salud de los menores de cinco años. De los 70 menores de 10 años evaluados (93,3\%), 34 tenían menos de cinco años. No se observaron déficits nutricionales y se registro sobrepeso en 11,4\% de los niños. La mayoría de los niños $(74,3 \%)$ vivía en domicilios con energía eléctrica, $95 \%$ en domicilios con letrina/retrete y un 52,9\% efectuaba deposiciones en fosa séptica. La realización de seis o más consultas de carácter prenatal fueron informadas por parte de un $82,4 \%$ de las madres de los menores de cinco años, y un 91,2\% comenzaron las consultas prenatales durante el primer trimestre de gestación. Entre las causas de internamientos hospitalarios en los últimos 12 meses (23,5\%), solamente una se debió a diarrea $y$ ninguna a causa de infecciones respiratorias. Se verificaron coberturas universales para la mayoría de las vacunas evaluadas. La inexistencia de déficits nutricionales entre los niños Pataxó puede estar asociada a las mejores condiciones de vivienda, saneamiento y cobertura de las acciones básicas respecto a salud infantil, cuando se comparan con las condiciones verificadas en la I Encuesta Nacional de Salud y Nutrición de los Pueblos Indígenas $y$ de otros estudios puntuales. Este trabajo pretende servir de apoyo para futuras discusiones y acciones que tengan por objetivo las mejoras en el estado nutricional infantil de los indígenas en Brasil.

Salud de Poblaciones Indígenas; Antropometría; Indios Sudamericanos; Estado Nutricional
Recebido em 21/Set/2017

Versão final reapresentada em 20/Jan/2018 Aprovado em 23/Mar/2018 